\section{Re: Laurence Albiges, Tom Powles, Michael Staehler, et al. Updated European Association of Urology Guidelines on Renal Cell Carcinoma: Immune Checkpoint Inhibition Is the New Backbone in First-line Treatment of Metastatic Clear-Cell Renal Cell Carcinoma. Eur Urol 2019;76:151-6}

\section{Active Surveillance in Metastatic Renal Cell Cancer: Missing a Chance for Cure?}

We read with great interest the updated European Association of Urology (EAU) guidelines on renal cell carcinoma (RCC) described by Albiges et al [1]. According to the EAU guidelines, immune checkpoint inhibition (ICI) is recommended as the "backbone" of first-line treatment for patients with clear-cell metastatic RCC (mRCC), including patients with a favourable risk score according to the International Metastatic Renal Cell Carcinoma Database Consortium. For these patients, the recommended first-line treatment is the combination of pembrolizumab and axitinib, which consists of ICI (ie, PD-1 inhibition) and a tyrosine kinase inhibitor targeting VEGFR. However, the optimal timing for initiation of first-line ICI combinations is not well defined, as active surveillance is still used, especially for mRCC patients with a limited tumour burden and few symptoms [2].

For decades, active surveillance has been the standard of care in a subset of mRCC patients. In the era of ICI, the question rises as to whether active surveillance is still reasonable as first-line management of mRCC. First, randomised clinical trials supporting active surveillance for mRCC patients are lacking [2]. Second, patients with a low tumour burden have a higher chance of tumour response during PD-1 blockade [3]. Third, among mRCC patients treated with first-line ICI combinations, complete response rates of up to $9 \%$ have been observed [4] which is unsurpassed for systemic therapy in mRCC. Fourth, tumour responses during PD-1 blockade appear durable at 5 years [5] and it is expected that these responses will persist thereafter, as has been reported for ipilimumab monotherapy. This long-term survival emphasises the curative potential of ICI. In order not to miss potential chances for cure, active surveillance in mRCC may be gradually abandoned from current clinical practice.

As ICI is moving to earlier stages of mRCC, including the favourable risk group, it is expected that an increasing number of mRCC patients will experience durable responses. In addition, an increasing number of $\mathrm{mRCC}$ patients will be exposed to potential (severe) toxicity. The optimal treatment duration of ICI for mRCC is currently not well defined. Although responses are durable after early discontinuation of ICI, the treatment is usually continued until disease progression. Therefore, rules for a safe stop of ICI need to be defined for mRCC patients with durable tumour responses.

In the era of first-line $\mathrm{ICI}$, we believe that active surveillance needs reconsideration and should be reserved for selected mRCC patients. Considering the pros and cons of active surveillance versus first-line ICI, shared decisionmaking is essential for treatment planning for mRCC patients, in particular for patients with a low tumour burden or minimal symptoms.

Conflicts of interest: Karlijn de Joode has nothing to disclose. Astrid A.M. van der Veldt serves on advisory boards for Roche, BMS, MSD, Ipsen, Pfizer, Pierre Fabre, Novartis, and Sanofi

\section{References}

[1] Albiges L, Powles T, Staehler M, et al. Updated European Association of Urology guidelines on renal cell carcinoma: immune checkpoint inhibition is the new backbone in first-line treatment of metastatic clear-cell renal cell carcinoma. Eur Urol 2019;76:151-6.

[2] Escudier B, Porta C, Schmidinger M, et al. Renal cell carcinoma: ESMO Clinical Practice Guidelines for diagnosis, treatment and follow-up. Ann Oncol 2019;30:706-20.

[3] Joseph RW, Elassaiss-Schaap J, Kefford R, et al. Baseline tumor size is an independent prognostic factor for overall survival in patients with melanoma treated with pembrolizumab. Clin Cancer Res 2018;24:4960-7.

[4] Motzer RJ, Tannir NM, McDermott DF, et al. Nivolumab plus ipilimumab versus sunitinib in advanced renal-cell carcinoma. $\mathrm{N}$ Engl J Med 2018;378:1277-90.

[5] Topalian SL, Hodi FS, Brahmer JR, et al. Five-year survival and correlates among patients with advanced melanoma, renal cell carcinoma, or non-small cell lung cancer treated with nivolumab. JAMA Oncol. In press. https://doi.org/10.1001/jamaoncol.2019.2187.

https://doi.org/10.1016/j.eururo.2019.09.030

0302-2838/C 2019 European Association of Urology. Published by Elsevier B.V. All rights reserved. 
Karlijn de Joode $\mathrm{e}^{\mathrm{a}, \mathrm{b}}$ Astrid A.M. van der Veldt ${ }^{\mathrm{a}, \mathrm{b}, *}$

${ }^{a}$ Department of Medical Oncology, Erasmus MC Cancer Institute, Rotterdam, The Netherlands

${ }^{\mathrm{b}}$ Department of Radiology \& Nuclear Medicine, Erasmus MC, Rotterdam,
${ }^{*}$ Corresponding author. Department of Medical Oncology, Erasmus MC Cancer Institute, Dr. Molewaterplein 40, 3015 GD Rotterdam, The Netherlands. Tel. +31 107040704. E-mail address: a.vanderveldt@erasmusmc.nl (A.A. van der Veldt).

The Netherlands 